\title{
¿BLINDANDO LA «FORTALEZA EUROPEA»? INTERESES, VALORES Y CAMBIOS JURÍDICOS EN LA POLITICA MIGRATORIA DE LA UNIÓN EUROPEA

\author{
PETRA BENDEL*
}

RESUMEN. La política migratoria comunitaria de la Unión Europea está entrando en un nuevo periodo. Este trabajo analiza las fallas cometidas y los logros alcanzados hasta el momento por la Unión Europea en su propósito de construir una Política Común de Inmigración y Asilo. También evalúa el nuevo Programa de La Haya aprobado por el Consejo Europeo en noviembre de 2004, que determinará las políticas migratorias europeas para los próximos cinco años. El artículo llega a la conclusión de que el nuevo programa queda atrás de las directrices plasmadas en su antecesor, el Programa de Tampere, con respecto a la prevención de los movimientos migratorios, la inmigración laboral y la integración de los inmigrantes. Ahora, el interés político previsto para los próximos cinco años se restringe al control y restricción de los movimientos migratorios.

PAlAbras ClAVE: política migratoria, inmigración laboral, fortaleza europea, Programa de La Haya.

ABStRACT. Immigration policy in the European Union is at the beginning a new era. This articles synthesizes and analyzes both the successes and failures of the EU in its efforts to build a common immigration and refugee policy. It also assesses the new Den Hague Program, passed by the European Council in november 2004, which will determine the new European immigration policies for the next five years. The article reaches the conclusion that the new program represents a step back compared to the Tampere Program in regard to migrant flow prevention, labor migration and immigrant integration.

KEYWORDS: immigrant policies, tampere program, Den Hague Program. 


\section{INTRODUCCIÓN}

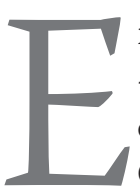

n mayo de 2004 se cumplió el plazo previsto en el Tratado de Ámsterdam —en vigor desde mayo de 1999- para que todas las regulaciones europeas conformaran una Política Comunitaria de Inmigración y Asilo. En 1999 el Consejo Europeo, integrado por los jefes de Estado, había acordado en Tampere un programa destinado a crear un «área de libertad, seguridad y justicia» (Alba y Leite, 2004: 6). Además de aprobar los procedimientos de asilo, ${ }^{1}$ el Consejo de la Unión Europea -o Consejo de Ministros, órgano clave para aprobar la legislación europea en el área de Justicia y Asuntos del Interior - votó las directrices y principios que configuran la ley marco europea cuyo contenido, según el Programa de Tampere, debe de ser adoptado en las respectivas legislaciones nacionales en un plazo determinado. En noviembre de 2004, el Consejo de la Unión Europea adaptó en Bruselas un programa nuevo, el Programa de La Haya, desarrollado durante la presidencia holandesa, que tendrá vigencia en los próximos cinco años en el área de Justicia y Asuntos del Interior. Además, en estos momentos estamos probablemente en vísperas de la ratificación del Tratado Constitucional Europeo, ${ }^{2}$ que reforzaría la tendencia introducida por el Programa de La Haya. Así pues, el momento actual parece ser el adecuado para detenernos y preguntar sobre cuáles son los logros, los errores y las perspectivas de la política comunitaria. En este artículo examinaremos, primero, hasta qué punto han adquirido rango comunitario - la transferencia de las competencias anteriormente nacionales al nivel supranacional de la Unión Europea - las políticas migratorias y en qué áreas, en cambio, se han mantenido las competencias de los Estados miembros. En segundo lugar, analizaremos cuáles son los contenidos de los reglamentos adoptados hasta ahora y de los previstos en el nuevo programa. En último lugar preguntaremos cómo se pueden evaluar estos elementos.

\section{POLÍTICAS MIGRATORIAS COMUNES EN LA UNIÓN EUROPEA: ¿CUÁLES SON LOS LOGROS?}

En Europa las políticas nacionales son ya impensables en algunas áreas importantes sin tomar en cuenta el nivel europeo. La Comunidad ha adquirido competencias enormes en la delicada política de inmigración y asilo. Con el Tratado de Ámsterdam, la legislación europea, especialmente la que se refiere a los visados y a la mayoría de los asuntos de asilo y refugio, está vinculada en términos jurídicos y de justicia en un esta-

\footnotetext{
${ }^{1}$ Procedimientos que todavía no se adoptan formalmente, pero que sí fueron acordados en abril de 2004.

${ }^{2}$ Durante la celebración del Consejo Europeo, el 18 y 19 de junio, los jefes de Estado y de Gobierno europeos llegaron a un acuerdo sobre la Constitución Europea. Se denomina aquí como «Tratado» porque se trata de un acuerdo intergubernamental, no de una verdadera Constitución (que presupone la existencia de un Estado; según mi entender, la Unión Europea es un sistema político, pero no un Estado). El Tratado Constitucional, que además de enunciar los valores en los que se fundamenta la Unión Europea y de dotarla de una personalidad jurídica, clarifica las funciones y competencias de los órganos e instituciones de la Unión. Antes de entrar en vigor, tiene que ser ratificado por todos los Estados miembros conforme a sus respectivas disposiciones constitucionales (aprobación parlamentaria en algunos países además de un referéndum o tan sólo por referéndum en otros países miembros).
} 
tuto superior al derecho de cada Estado. En los últimos cinco años, el poder legislativo, en este ámbito especialmente delicado, correspondía a la Comisión Europea, que lo compartía con los Estados miembros. El Parlamento Europeo tan sólo debía ser consultado - muchas veces, simplemente ignorado- mientras que el poder de decisión correspondía al Consejo de Ministros.

La toma de decisiones se topó con el poder de veto que de hecho poseía cada uno de los quince ministros nacionales de Justicia y Asuntos del Interior en el Consejo de la Unión Europea, ya que cada directriz tenía que aprobarse por unanimidad. Ésta constituía, en muchos casos, una gran barrera para que se acordaran leyes europeas, dada la heterogeneidad de intereses, valores y tradiciones en el trato a los inmigrantes. Mientras que algunos Estados miembros (como Alemania y, más tarde, Reino Unido) se consideraban en desventaja por el gran número de refugiados que acogieron, otros (España, Portugal, Grecia e Italia) señalaban sus largas y desprotegidas fronteras. Mientras Reino Unido, Países Bajos y los países escandinavos poseen una tradición liberal y multicultural en el trato a los inmigrantes - que en algunos de estos países está cambiando últimamente de manera radical-, otros empiezan a crear una política de integración, como Alemania - a pesar de haber atraído un gran número de trabajadores extranjeros hace décadas- y como los países de tradición más bien emigrante del sur europeo - España, Italia, Portugal y Grecia-.

Hay que reconocer que a pesar de esta dificultad institucional, el Consejo de Ministros aprobó una serie de leyes europeas que ahora regulan el ámbito de la inmigración y asilo político en todos los países miembros de la Unión, aunque todavía debe de investigarse cuáles fueron los intereses y valores y cuáles los actores que finalmente se impusieron en las distintas negociaciones. El poder de veto que poseían los ministros nacionales se ha ido reduciendo paulatinamente, al tiempo en que se han extendido las competencias del Parlamento Europeo. Según el artículo 67 del Tratado Constitucional Europeo, después de que ha vencido el periodo transitorio, el 1 de mayo de 2004, el Consejo debería votar para cambiar el reglamento concerniente a la toma de decisiones. El Consejo, en un futuro próximo, podría llegar a votar por mayoría calificada, procedimiento que consideramos muy aconsejable después de que la Unión Europea aumentó su membresía a 25 Estados y a un número igual de ministros en el Consejo. ${ }^{3}$ El Parlamento Europeo, que hasta ahora sólo debía de ser «consultado», podría entonces adquirir mayores competencias a través del llamado procedimiento de codecisión. Este procedimiento le otorga un poder legislativo mucho más amplio al Parlamento Europeo (que al fin y al cabo sigue siendo el único órgano directamente elegido y legitimado de la Unión Europea), que lo comparte con el Consejo de la Unión Europea. El Programa de La Haya llama a aceptar estos reglamentos de toma de decisiones, establecidos en el Tratado de Niza, a más tardar el 1 de abril de 2005.

\footnotetext{
${ }^{3}$ La mayoría calificada, que pasará a ser la norma general para la toma de decisiones en el Consejo de Ministros, fue objeto de la mayor discusión en la Convención Europea, encargada de elaborar la Constitución. La fórmula finalmente definida se basa en el principio de doble mayoría con los rangos siguientes: un mínimo del 55\% de los Estados miembros que incluya por lo menos 15 de ellos y represente a Estados miembros que reúnan como mínimo el $65 \%$ de la poblacion. Una minoría de bloqueo, el 35\% de la población, debe sumar por lo menos cuatro Estados miembros, sin la cual se considera adoptada la decisión. Este sistema no funcionará hasta el 1 de noviembre de 2009. La unanimidad seguirá siendo la norma por lo que se refiere a temas como la fiscalidad y parcialmente temas de la política exterior, de seguridad común y de política social.
} 
La propuesta del Tratado Constitucional también prevé recurrir a los mecanismos de mayoría calificada y codecisión para todas las medidas que la Unión Europea tome en materia de inmigración y asilo, excepto en la inmigración legal.

Es precisamente la migración legal el ámbito en el cual algunos Estados miembros se aferran celosamente a sus competencias nacionales. Mientras Reino Unido, Irlanda y Dinamarca suelen hacer uso del opt-out en la política de inmigración, asilo y derecho civil (Peers, 2004), Alemania y sus estados federados — que pelearon «en casa» por un nuevo derecho migratorio nacional, finalmente aprobado en 2004, e intentan «proteger» sus mercados laborales- defienden su derecho a mantener la competencia nacional en todo lo que se refiera al derecho laboral y, con ello, el derecho a «vetar» las propuestas de la Comisión en el Consejo. Por eso, el Programa de La Haya conserva el voto unánime en el Consejo de la Unión Europea en algunos ámbitos claves así como los derechos restringidos del Parlamento que tan sólo debe de ser consultado. Esto vale para los derechos de inmigrantes residentes con larga duración y el derecho de libre circulación para nacionales de terceros países con hasta tres meses de permanencia, y para otras medidas como la abolición de controles fronterizos internos entre los Estados miembros, los controles fronterizos externos, medidas contra la inmigración «ilegal», el burden sharing en el régimen de asilo y el derecho familiar dentro del derecho civil. Alemania y sus Länder consiguieron también imponer esta posición dentro de la Convención Europea. Se introdujo en el Tratado Constitucional la competencia nacional y no europea para todos aquellos reglamentos que a la migración económica se refieren. Más aún, el acceso al mercado laboral para nacionales de terceros países sigue constituyendo un tema muy delicado, incluso en palabras de la misma Comisión Europea - defensora de una mayor comunitarización de estas políticas- sólo puede ser europeizado muy paulatinamente.

\section{EL CONTENIDO DE LAS POLÍTICAS COMUNES: ¿QUÉ METAS PREVALECEN?}

La política migratoria, según mi entender, puede aspirar a lograr metas distintas, incluso contradictorias, como i) la restricción y control de la inmigración, ii) la protección de los refugiados, iii) la prevención de los movimientos de refugiados, iv) la integración de los inmigrantes y $v$ ) la atracción de grupos especiales de inmigrantes.

Si aceptamos esta sistematización, tenemos que reconocer que las políticas comunes europeas que se han realizado hasta ahora tienden a concentrarse excesivamente en el uso de instrumentos de control y restricción de la migración, en el combate contra la inmigración irregular y en la política de expulsión y repatriación. A veces, y con razón, se ha hablado de que las políticas migratorias se reducen al menor número de Estados-nación. El burden sharing entre los Estados miembros que aceptan asilados y refugiados a través del Fondo Europeo de Refugiados —renovado para el periodo 2005-2010- y la determinación del Estado que se considera responsable del examen de una solicitud de asilo siempre han estado al tope en la agenda de las políticas migratorias comunes desde principios de los años noventa. El Consejo de Ministros introdujo un sistema de información y control de las fronteras en Schengen (SIS I y SIS II) y un sistema de informatización de visados (VIS). Se adoptó un sistema electrónico dactiloscópico (EURODAC) para determinar qué Estado tiene la responsa- 
bilidad de tratar una solicitud de asilo, y un sistema de archivo de imágenes (FADO) con el fin de combatir la inmigración «ilegal». El actual Programa de La Haya quiere conectar estrechamente los tres sistemas SIS II, VIS y EURODAC. Se han ajustado los sistemas de visados, se han armonizado las políticas de repatriación de los expulsados, así como aquellas medidas que se refieren al control de las fronteras exteriores de la Unión. Asimismo, el Consejo ha adoptado medidas amplias contra la inmigración «ilegal $»^{4}$ y contra el tráfico de personas. ${ }^{5}$

Este acento predominante en la restricción y control de la inmigración demuestra que en la Unión Europea se percibe la inmigración mayormente como un problema de seguridad. Esto se reforzó, por supuesto, después de los acontecimientos terroristas del 11 de septiembre de 2001 en Nueva York y del 11 de marzo de 2004 en Madrid. El Programa de La Haya lo subraya otra vez: el combate al terrorismo es la primera meta que el programa menciona como centro de la política comunitaria de Justicia y Asuntos del Interior. Dentro de las políticas de inmigración y asilo, que forman parte de esta área, el tema de seguridad sobresale en el control fronterizo, el «combate contra la inmigración ilegal»y en muchas otras directrices dadas a conocer por los jefes de Estado. Con el fin de aumentar la seguridad, el Consejo Europeo exige a los órganos europeos y a los Estados miembros continuar sus esfuerzos para integrar, «sin demora», la identificación biométrica en los documentos de viaje, permisos de residencia, pasaportes de los ciudadanos europeos y de preparar los estándares mínimos para las tarjetas de identidad nacional. Asimismo, el intercambio de la información sobre la vigencia de la ley es otro elemento importante de las políticas comunes. El control y la vigilancia de las fronteras son, por supuesto, competencias que corresponden todavía a los Estados miembros. No obstante, se piensa crear una asistencia fronteriza especializada, European Agency for the Management of Operational Cooperation at the External Borders, para establecerse en mayo de 2005. El Consejo Europeo invita incluso a estudiar la viabilidad de crear una guardia fronteriza europea. La seguridad, por supuesto, es una meta importante, legítima y necesaria en materia migratoria.

Sin embargo, una crítica de las políticas llevadas a cabo hasta el momento debe de centrarse en el hecho de que las demás metas de la política migratoria han quedado relativamente sin desarrollar. Son pocos los reglamentos comunes que se refieren a la protección de los refugiados, la prevención de los movimientos de refugiados, la integración de los inmigrantes (relacionado con el tema de seguridad) y la atracción de los inmigrantes que vienen con fines económicos. Los reglamentos que se aprobaron en estos ámbitos son, en su gran mayoría, relativamente rígidos en su contenido.

Es cierto que se definieron las normas mínimas para acoger a los solicitantes

\footnotetext{
${ }^{4}$ Prefiero este término entre comillas, usado en los respectivos documentos de la Unión Europea, y usar el término de inmigrantes «irregulares» o «sin papeles». El término «ilegal», proveniente del derecho, me parece estigmatizar $y$, posiblemente, criminalizar socialmente al inmigrante. No existe la ilegalidad a priori. Como todos los términos del derecho, el de la ilegalidad está sujeto a un cambio sociohistórico. Cambiando los fundamentos legales, ciertas categorías de inmigrantes, que en un momento histórico se consideraban «legales», pueden llegar a ser «ilegales». Además, muchos inmigrantes ni siquiera saben cuáles de las múltiples normas jurídicas sancionan su cruce de fronteras hacia la Unión Europea.

${ }^{5}$ Esta lista se podría alargar. Para ver una lista detallada de la legislación europea compárese el scoreboard de la Unión que se encuentra en 〈http://europa.eu.int/comm/justice_home〉. El Programa de La Haya invita a la Comisión para presentar un programa parecido al Consejo una vez al año, hasta ahora cada seis meses, para poder seguir la implementación del programa.
} 
de asilo (2003/9/CE), para la protección temporal en caso de afluencia masiva de desplazados (2001/55/CE), para el procedimiento de concesión y retirada del estatuto de refugiado. No obstante, las formas de protección subsidiaria para aquellas personas no incluidas en la Convención de Refugiados de 1951, pero que necesitan protección internacional, se quedaron muy por detrás de las expectativas que tenían las Organizaciones no Gubernamentales (ONG) preocupadas por el destino de los refugiados y desplazados. Además, la directriz que garantizaría normas mínimas para el procedimiento de asilo es actualmente blanco de críticas por sus bajos estándares en materia de derechos humanos. Dicha directriz se acordó a tiempo en el plano político, pero no así en el plano formal-jurídico. El nuevo Programa de La Haya invita incluso a estudiar la posibilidad de tratar comunitariamente las solicitudes de asilo fuera de la Unión Europea, pero el texto no concretiza qué podría suponer este procedimiento (Steve Peers, 2004a).

Respecto a la prevención de los movimientos de refugiados, la Unión Europea tampoco ha brillado por su actividad. A pesar de que en la retórica uno de los pilares fundamentales de la política migratoria comunitaria consiste en la cooperación con los países de origen, ésta cooperación también se ha limitado a grandes líneas de control. La colaboración con «terceros países» (es decir, países no miembros de la Unión), según el Programa de La Haya, es, sobre todo, establecida para aumentar la capacidad de estos países en su gestión migratoria y en la protección de refugiados, prevenir y combatir la inmigración ilegal, informar sobre los canales legales para la inmigración, resolver la situación de los refugiados dándoles acceso a soluciones durables, construir mayor capacidad para controlar sus fronteras, mejorar la seguridad de los documentos y resolver el problema del retorno (Consejo Europeo, 2004). Al menos, el Consejo apoya el comunicado aprobado por la Comisión Europea en el sentido de mejorar el acceso de los refugiados a soluciones duraderas e invita a la misma comisión a desarrollar programas regionales con los países de origen.

Enfocando el tema de la integración de los inmigrantes que ya están viviendo en la Unión Europea, el principio de la no discriminación, tal y como lo prevé el artículo 13 del Tratado de Ámsterdam, otorga el derecho al Consejo de tomar acciones apropiadas para combatir la discriminación por razones de sexo, raza o etnia, religión u opinión, defecto físico, edad u orientación sexual. Con base en esta norma, el Consejo de la Unión Europea ha adoptado una directriz sobre la no discriminación racial (2000/43/CE) y otra sobre la no discriminación profesional (2000/78/CE) — un hecho que sorprende a muchos observadores, ya que estas directrices son de carácter bastante liberal-. En ellas se impuso la tradición británica sobre la de otros países con mayor relevancia en materia de inmigración (Geddes y Guiraudon, 2003). ${ }^{6}$ A pesar de la gran importancia que tienen estos acuerdos, también es cierto que muchos Estados miembros todavía no han implementado estos principios de manera completa, aunque el plazo para convertir la directriz en ley nacional ya venció a finales de 2003 (Jenaro Tejada, Carles-Berkowitz y Chopin, 2002; Nickel, Coomber y Bell, 2003). La Comisión Europea ha amenazado ya con sancionar la no aplicación de las leyes europeas. La reunificación familiar, uno de los aspectos clave de la política de integración y aún uno de los factores más importantes de atracción de inmigrantes, tan sólo fue regulado después de duros debates que duraron cuatro años. La directriz que resultó

${ }^{6}$ Reino Unido tiene el opt-out en muchos aspectos, y no participó en el Acervo de Schengen. 
de estas negociaciones (2003/86/CE) es ahora tratada por la Corte Europea de Justicia (CEJ), puesto que el Parlamento Europeo entabló una acción judicial por violación de su derecho a examinar la última versión de la directriz y a presentar sus comentarios y observaciones. Detrás de esta acción por anulación de la directriz se esconde la convicción de que muchas medidas que aquí se formulan violan la Convención Europea de Derechos Humanos, especialmente aquéllas que se refieren al derecho de los niños. Mientras tanto, los derechos de los residentes de larga duración fueron aprobados (2003/109/CE) en el Consejo. Esta directriz está destinada a equiparar los derechos de los nacionales de terceros países con aquellos de los ciudadanos europeos (Konstakopoulou, 2001). También es cierto que la Comisión Europea ha llamado a los Estados miembros a cooperar en mayor medida para garantizar la integración de sus inmigrantes. Además, en 2003 lanzó un comunicado que presenta un marco ambicioso, complejo y coherente para mejorar la integración de los inmigrantes de manera comunitaria (Commission of the Euroepan Communities, 2003). Sin embargo, el progreso para seguir estas recomendaciones ha sido sumamente lento y a ratos, incluso, completamente bloqueado. Al menos se inició una serie de proyectos piloto en materia de integración, se apoyó a redes de ONG y la trasferencia de información y «buenas prácticas». La integración de nacionales de terceros países en la sociedad representa, por supuesto, una tarea muy compleja que incluye la integración a los mercados laborales, la educación y el conocimiento de la lengua, la vivienda, servicios sanitarios y sociales, la nacionalidad y el respeto de la diversidad social. Todas estas cuestiones, empero, aún forma parte del ámbito de competencia de los Estados miembros. El Programa de La Haya insiste en la necesidad de intercambiar información y experiencias, pero no toca las competencias nacionales.

Es en el ámbito de la inmigración legal en el cual los países miembros mantienen férreamente sus competencias, especialmente con respecto a la atracción de grupos especiales de inmigrantes. Por otro lado, la Comisión Europea también presentó una propuesta interesante sobre eventuales políticas comunes en esta área (COM 2003/336 final), que enfatiza la importancia de atraer e integrar a inmigrantes de terceros países en los mercados laborales europeos. Si bien la Comisión reconoce abiertamente que la inmigración tiene mucha importancia económica y demográfica para ciertos sectores y regiones de la Unión Europea, y a pesar de que los mismos Estados miembros ven la necesidad de fomentar la inmigración para cubrir necesidades en ciertas profesiones de sus mercados laborales, aún no disponemos de un mercado laboral común en la Unión y, por lo tanto, tampoco compartimos intereses comunes para aumentar las competencias de la Unión en ésta área. ${ }^{7}$ La directriz propuesta en julio de 2001 por la Comisión Europea (COM 2002/386), sobre las definiciones, criterios y procedimientos para los nacionales de terceros países que trabajan en la Unión Europea, había respetado, en un principio, la discrecionalidad de los Estados miembros para limitar la inmigración laboral. En su propuesta, preveía la posibilidad de atraer a inmigrantes de manera rápida y flexible, según las respectivas necesidades económicas

\footnotetext{
Claro está que la migración intracomunitaria desde los nuevos países miembros de la Unión Europea puede ayudar a amortiguar los efectos de las distorsiones demográficas y las necesidades profesionales del futuro. Sin embargo, se pronostica que la migración desde países como Polonia, República Checa, Hungría, Slovenia y Estonia tendrá más bien un carácter temporal que duradero.
} 
y demográficas. Debido, sobre todo, a la oposición alemana, esta propuesta ya no ha sido debatida en el Consejo de Ministros desde octubre de 2002. La Comisión debería haber presentado un green paper sobre la migración laboral en octubre de 2004, pero aún no lo hace. En el Programa de La Haya, el Consejo Europeo hizo alguna confesión de dientes para afuera con respecto a la migración laboral en una economía europea basada en el conocimiento, para avanzar en el desarrollo económico que contribuya a la implementación de la Estrategia de Lisboa. Pero al mismo tiempo, falla al mencionar los principios consignados por el Consejo Europeo de Tampere, como el tratamiento de los nacionales de terceros países comparable al de los nacionales, el tratamiento igualitario de los residentes de largo plazo similar al de los nacionales (Peers, 2004a). También subraya que la «determinación de la cantidad de inmigrantes laborales aceptables es competencia de los Estados-nación». Tomando en cuenta el resultado de los debates sobre el green paper acerca de la inmigración laboral, las buenas prácticas en los Estados miembros y su relevancia para implementar la Estrategia de Lisboa, el Consejo también invita a la Comisión a presentar antes de que termine 2005 un plan político sobre la migración legal, que incluya los procedimientos de admisión capaces de responder rápidamente a las demandas fluctuantes de la inmigración laboral (Conseil Européen, 2004). Esto podría resumir al menos algunos aspectos de la directriz «Congelada» sobre inmigración laboral, pero es evidente que esta política no se considera como prioritaria, ya que el programa no fija ningún plazo para terminarla.

\section{¿CÓMO SE EVALÚAN LOS CONTENIDOS?}

Como hemos podido observar, el control fronterizo, las sanciones contra el tráfico de seres humanos y las políticas de retorno son todavía las piedras angulares de la muralla de la «fortaleza europea», una fortaleza que parece tentada a extender sus murallas más allá de sus propias fronteras. El Programa de La Haya ha restringido las prioridades nuevamente a los aspectos de seguridad, sobre todo en aquellas medidas que refuerzan la restricción y el control. Con su Programa de La Haya, el Consejo Europeo se queda atrás del programa antecesor, el Programa de Tampere. Aquel programa al menos aspiró a regular los movimientos de refugiados, su protección y la integración de los inmigrantes en la Unión Europea. Sin embargo, si tomamos en cuenta los cinco criterios arriba desarrollados, éstos aspectos seguramente no pueden ser suficientes para construir el acceso polifacético y moderno que exige la inmigración en la Unión Europea.

Sin duda alguna, el tema de la seguridad tiene gran importancia en materia migratoria, y más a la luz de los acontecimientos recientes en distintos países europeos. Primero, el aspecto de la seguridad no puede limitarse a medidas de restricción y control. Se queda cojo si elude el tema central de la integración. Segundo, pienso que la política comunitaria inmigratoria aún no hace suficiente justicia a un fenómeno tan complejo como el inmigratorio. Éste, como todos sabemos, se compone de grupos migratorios muy distintos, con necesidades y prioridades diferentes y que exigen desarrollar políticas diversas y flexibles. Las políticas migratorias deben de corresponder a necesidades de los países de la misma Unión Europea, necesidades que se derivan de los problemas del mercado laboral y de la evolución demográfica. Tercero, las políticas migratorias tienen que hacer justicia a los valores que ha reivindicado la Unión Europea, que se autodefine, en el nuevo Tratado Constitucional, como una Unión basada 
en «la dignidad humana, la libertad, la democracia, la igualdad, el Estado de derecho y la vigencia de los derechos humanos». Es ahí donde están los grandes desafíos de la política migratoria futura de la Unión. Sin esos principios, el mote de «fortaleza europea» seguirá teniendo su justificación.

\section{REFERENCIAS}

AlbA, F. y P. Leite (2004), «Políticas migratorias después del 11 de septiembre: los casos del TLCAN y la UE», en Migración y Desarrollo, núm. 2, pp. 4-20.

Ahnfelt, E. y J. From (2001), «Policy on Justice and Home Affairs: From high to low politics», en Svein S. Andersen y Kjell A. Eliassen (eds.), Making Policy in Europe, London, pp. 140-166.

Angenendt, S. (ed.) (1999), Asylum and Integration Policies in the European Union, Deutsche Gesellschaft für Auswärtige Politik/Forschungsinstitut, Bonn.

Alink, F., A. Boin y P. Hart (2001), «Institutional crises and reforms in policy sectors: the case of asylum policy in Europe», en Journal of European Public Policy, núm. 8, abril, pp. 286-306.

Andersen, Svein S. y Kjell A. Eliassen (2001) (eds.), Making Policy in Europe, London.

Appleyard, R. (2001), «International Migration Policies: 1950-2000», en International Migration, núm. 39, pp. 7-20.

Arango, J. (1992), «Los dilemas de las políticas de inmigración en Europa», en Cuenta y Razón, núm. 73.

Bernstein, A. et al. (eds.) (1999), Migration and refugee policies: an overview, London. Bloch, A. (ed.) (1999), Refugees, Citizenship and Social Policy in Europe, London.

BöcKer, A. y T. Havinga (1998), «Asylum applications in the European Union: patterns and trends and the effects of policy measures», en The Journal of Refugee Studies, núm. 11, septiembre, pp. 245-266.

Bosswell, C. (2002), «Explaining European Public Policy Responses to Asylum and Migration», trabajo presentado en la UNHCR-WIDER Conference on Poverty, International Migration and Asylum, Helsinki, septiembre. (2003), European Migration Policies in Flux. Changing Patterns of Inclusion and Exclusion, London.

Cesarani, D. y M. Fulbrook (eds.) (1996), Citizenship, Nationality and Migration in Europe, London/New York.

Coleman, D.A. (1999), «Europe under migration pressure: some facts on immigration», en E. Ucarer, The coming of an era of human uprootedness: a global challenge, London/Washington.

Consejo Europeo de Bruselas (2004), «Conclusiones de la Presidencia», noviembre.

Cornelius, W.A., P.L. Martin y J.F. Hollifield (eds.) (1994) Controlling Immigration. A Global Perspective, Stanford, CA.

Delgado Godoy, L. (2002), «La inmigración en Europa: realidades y políticas, Unidad de Políticas Comparadas (CSIC)», documento de trabajo, Universidad Rey Juan Carlos, en 〈http://www.iesam.csic.es/doctrab2/dt-0218.pdf〉.

Dell'Olio, F. (2001), «Integration patters in the EU: an analysis of different approaches towards the process of immigrants' integration», documento presentado 
en la $29^{\text {th }}$ Joint Sessions of Workshops ECPR, abril, Grenoble, France, Immigration, Integration and European Union: Institutional Practices and Normative Challenges.

Dittgen, H. (1999), «Immigration Control: Some Observations on National Traditions», en A. Schulte y D. Thränhardt (eds.), en International Migration and Liberal Democracies. Internationale Migration und freiheitliche Demokratien. Jahrbuch Migration 1999/2000, Münster, pp. 77-86.

European Commission (2001), The Role of Immigrants in the Labour Market, survey among the Member States.

European Council on Refugees and Exiles (2001) The Promise of Protection: Progress towards an European Asylum Policy since the Tampere Summit 1999, Brussels, noviembre.

FAVELL, A. (1998), «The Europeanisation of immigration politics», en European Integration online Papers, núm. 10, en 〈http://eiop.or.at/eiop/texte/1998-010a.htm〉.

Favell, A. y A. Geddes (1999), «European Integration, Immigration and the Nation State: Institutionalising Transnational Political Action?», en Robert Schumann, Working Papers, Centre for Advanced Studies, núm. 99, European University Institute.

Geddes, A. (2003), «Still Beyond Fortress Europe? Patterns and Pathways in Eu Migration Policy», en Queen's Papers on Europeanisation, núm. 4.

Geddes, A. y V. Guiraudon (2003), «Anti-discrimination Policy: The Emergence of a EU Policy Paradigm amidst Contrasted National Models», en Paper for the Workshop Opening the Black Box: Europeanisation, Discourse and Policy Change, Oxford, England.

Guild, E. (ed.) (1996), The Developing Immigration and Asylum Policies of the European Union. Adopted Conventions, Resolutions, Recommendations, Decisions and Conclusions, The Hague, London/Boston.

Guiraudon, V. (2001), «The EU 〈garbage can»: Accounting for policy developments in the immigration domain», paper presented at the Conference of the European Community Studies Association in the panel «Immigration and the Problems of Incomplete European Integration», Madison Wisconsin, mayo-junio. (2003), «The constitution of a European immigration policy domain: a political sociology approach», en Journal of European Public Policy, núm. 10, abril, pp. 263-282.

Hailbronner, K. (1998), «European Immigration and Asylum Law under the Amsterdam Treaty», en Common Market Law Review, núm. 35, pp. 2047-2067.

Huysmans, J. (2000), «Contested community: migration and the question of the political in the EU», en Morten Kelstrup y Miachel C. Williams (eds.), International relations theory and the politics of European integration: power, security, and community, New York, pp. 149-170.

Jenaro Tejada, E., I. Carles-Berkowitz y I. Chopin (2002), Ohne Unterschied! Umsetzung der Richtlinie zur Anwendung des Gleichbehandlungsgrundsatzes-Überblick und Vorschläge, Brussels.

Joppкe, C. (1997), «Why liberal states accept unwanted immigration», en World politics, núm. 50, Princeton, NJ, pp. 266-293.

KINZ, J. (2001), Labour mobility and EU enlargement: a review of current trends and debates, Brussels. 
Koslowski, R. (1998), «European Union Migration Regimes, Established and Emergent», en Christian Joppke (ed.), Challenge to the Nation-State. Immigration in Western Europe and the United States, Oxford, pp. 153-188.

Lavenex, S. (2001), The Europeanisation of Refugee Policies. Between human rights and internal security, Aldershot U.A.

LEVy, C. (1999), «Europan asylum and refugee policy after the Treaty of Amsterdam: the birth of a new regime?», en Alice Bloch (ed.), Refugees, Citizenship and Social Policy in Europe, London, pp. 12-49.

LuCAS, J. (1992), Europa: ¿convivir con la diferencia?, Tecnos, Madrid.

Malgesini, G. y C. Giménez (2000), Guía de conceptos sobre migraciones, racismo e interculturalidad, Madrid.

Monar, J. (1997), «Comprehensive Migration Policy: The Main Elements and Options», en K. Heilbronner, D. Martin y H. Motomura (eds.), Immigration admissions: the search for workable policies in Germany and United States, Berghan, pp. 53-78.

Kostakopoulou, T. (2001), «Invisible Citizens: Long-term Resident Third Resident Third Country Nationals in the EU and their Struggle for Recognition», en R. Bellamy y A. Warleigh (eds.), Citizenship and Governance in the EU, LondonNew York, pp. 180-205.

(2002), «Integration Non-EU Migrants in the European Union: Ambivalent Legacies and Mutating Paradigms», en Columbia Journal of European Law, núm. 8, pp. 181-201.

MArtín y Pérez de Nanclares, José (2002), La inmigración y el asilo en la Unión Europea. Hacia un nuevo espacio de libertad, Seguridad y Justicia, Madrid.

MuUs, P.J. y E.W. van Dam (1998), Comparative research on international migration and international migration policy: migration from the Maghreb and Turkey to the European Union, and from Mexico, Guatemala and El Salvador to the United States, European Commission, Luxembourg.

Nickel, R., A. Coomber, M. Bell et al. (2003), Racism as a crime (N.Y.), European Strategies to combat racism and xenophobia as a crime, Brussels.

Niessen, J. (2001), «Overlapping Interests and Conflicting Agendas: The Knocking into Shape of EU Immigration Policies», en European Journal of Migration and Law, núm. 3, pp. 419-434.

Peers, S. (2002), «Key Legislative Developments on Migration in the European Union», en European Journal of Migration and Law, núm. 4, pp. 339-367. (2004), «Statewatch Briefing. Vetoes, Opt-outs, and EU Immigration and Asylum Law», prepared for statewatch by professor Steve Peers, University of Essex, revised version, en http://www.statewatch.org/news/2004/nov/eu-immig-optouts2.pdf).

(2004a), Statewatch: The «Hague Programme». Annotation of final version, annotated by professor Steve Peers, University of Essex, en 〈http://www.statewatch.org/news/2004/nov/hague-programme-final.pdf>.

Pro, Asyl (2002), Der lange Weg zu einem europäischen Asylrecht, materialien, Frankfurt, septiembre.

Ramos, J.A. et al. (1998), La política para la integración social de los inmigrantes: una perspectiva intergubernamental, Instituto Universitario Ortega y Gasset, Madrid. 
Salt, J. (2001), «Current Trends in International Migration in Europe», en Council of Europe, CDMG, núm. 33, noviembre.

SARtori, G. (2001), La sociedad multiétnica. Pluralismo, multiculturalismo y extranjeros, Madrid.

Stetter, S. (2000), «Regulating migration: authority delegation in justice and home affairs», en Journal of European Public Policy, núm. 7, marzo, pp. 80-103.

ThräNhARDT, D. (ed.) (1996), Europe-a new immigration continent: policies and politics in comparative perspective, Münster. 